\title{
Semiclassical theory of ballistic transport through chaotic cavities with spin-orbit interaction
}

\author{
Jens Bolte* \\ Institut für Theoretische Physik, Universität Ulm, Albert-Einstein-Allee 11, D-89069 Ulm, Germany \\ Daniel Waltner ${ }^{\dagger}$ \\ Institut für Theoretische Physik, Universität Regensburg, D-93040 Regensburg, Germany \\ (Received 20 April 2007; revised manuscript received 28 June 2007; published 16 August 2007)
}

\begin{abstract}
We investigate the influence of spin-orbit interaction on ballistic transport through chaotic cavities by using semiclassical methods. Our approach is based on the Landauer formalism [IBM J. Res. Dev. 1, 223 (1957); 32, 306 (1988)] and the Fisher-Lee relations [Phys. Rev. B 23, 6851 (1981)], appropriately generalized to spin-orbit interaction, and a semiclassical representation of Green's functions. We calculate conductance coefficients by exploiting ergodicity and mixing of suitably combined classical spin-orbit dynamics, and making use of the Sieber-Richter method [Phys. Scr., T T90, 128 (2001); Phys. Rev. Lett. 89, 206801 (2002)] and its most recent extensions. That way, we obtain weak antilocalization and confirm previous results obtained in the symplectic ensemble of random matrix theory.
\end{abstract}

DOI: 10.1103/PhysRevB.76.075330

PACS number(s): 73.23.-b, 71.70.Ej, 72.15.Rn, 03.65.Sq

\section{INTRODUCTION}

Ballistic transport through chaotic cavities realized as quantum dots in semiconductor heterostructures has been a central issue in mesoscopic physics for many years. The universal transport properties observed in this context can be described on a phenomenological level by random matrix theory $^{1}$ (RMT). The same applies to disordered systems, where averages over impurities can be shown to be equivalent to random matrix averages. This not being possible for individual, clean cavities, theoretical explanations of the RMT connection have been provided making use of semiclassical methods, which are based on the Landauer formalism ${ }^{2,3}$ and semiclassical representations of Green's functions. This approach ${ }^{4}$ leads to questions that are closely analogous to problems arising in semiclassical explanations of universal spectral correlations in classically chaotic quantum systems. Recent progress in the latter context is based on the seminal work of Sieber and Richter ${ }^{5}$ and its extensions. ${ }^{6-8}$ This method has been adapted ${ }^{9-12}$ to be able to successfully explain conductance coefficients, including the effect of weak localization, i.e., a decrease of conductance at zero magnetic field. Further studies have been devoted to analyses of the universality of conductance fluctuations, ${ }^{12,13}$ of shot noise, ${ }^{12,14-16}$ and of general higher moments. ${ }^{17}$ (For an overview see, e.g., Ref. 12.)

In the work mentioned, transport properties were considered for ballistic, nonrelativistic electrons, neglecting their spin. In the emerging field of semiconductor based spin electronics ${ }^{18}$ (spintronics), however, one requires an efficient control of the spin dynamics associated with electrons in nonmagnetic semiconductors. This purpose calls for studies of transport properties in systems with sufficiently strong spin-orbit couplings, i.e., where the dwell time is much larger than the spin relaxation time. In contrast to previous theories neglecting the spin, there one would expect appropriate classical spin-orbit dynamics to produce weak antilocalization, i.e., an enhancement of the conductance at zero magnetic field. This prediction is also obtained on the phenomenological level provided by RMT, where a half-integer spin requires the symplectic, as opposed to the orthogonal, circular ensemble. On this ground, one expects universal conductance fluctuations and other transport properties also to be affected by the presence of spin-orbit interactions. ${ }^{1,19} \mathrm{~A}$ first semiclassical approach ${ }^{20}$ to these questions employs the semiclassical representation of the Green's function in spinorbit coupling systems derived in Ref. 21 and considers the first order of the semiclassical Sieber-Richter expansion. It, moreover, assumes a randomization of spin states, which is shown to be responsible for weak antilocalization.

In this paper, our goal is to extend the results of Ref. 20 to all orders of the Sieber-Richter expansion and to base the semiclassical estimates entirely on dynamical properties of suitably combined classical spin-orbit dynamics. ${ }^{22}$ These then replace the randomization hypothesis of spin states made in the analytic part of Ref. 20. In order to determine the spin contribution to transmission amplitudes, we closely follow an analogous calculation introduced in the context of semiclassical explanations of spectral correlations in quantum graphs with spin-orbit couplings. ${ }^{23,24}$ We also comment on shot noise and on the variance of conductance fluctuations.

As our model, we consider a two dimensional cavity with two straight, semi-infinite leads with hard walls. Apart from boundary reflections, particles with mass $m$, charge $e$, and spin $s$ move freely within the leads and are subjected to a magnetic field and to spin-orbit interactions inside the cavity. Although the relevant case of electrons enforces the spin to be $s=1 / 2$, we deliberately allow for general spin $s$. Below, this will allow us to point out characteristic differences between integer and half-integer spins. The Hamiltonian governing the dynamics in the cavity reads

$$
\hat{H}=\frac{1}{2 m}\left[\hat{\mathbf{p}}-\frac{e}{c} \mathbf{A}(\hat{\mathbf{x}})\right]^{2}+\hat{\mathbf{s}} \cdot \mathbf{C}(\hat{\mathbf{x}}, \hat{\mathbf{p}})
$$

Here, $\mathbf{A}$ is the vector potential for an external magnetic field and $\mathbf{C}$ contains all couplings of the translational degrees of freedom to the spin operator $\hat{\mathbf{s}}$. This may be a Zeeman cou- 
pling, or any type of spin-orbit interactions, including Rashba and Dresselhaus couplings. Moreover, in order to model the hard walls, we require Dirichlet conditions at the boundaries of the cavity and of the leads.

The paper is organized as follows: Section II is devoted to a generalization of the Landauer formalism and the FisherLee relations to systems with spin-orbit interaction. Then, we present semiclassical representations of $S$-matrix elements in that case. In Sec. III, we first introduce ergodicity and mixing conditions that include a classical spin-orbit interaction. This is followed by our calculation of the conductance in two ways: in the configuration-space and in the phase-space approach. In Secs. IV and V, we then outline how our approach can be extended to calculate shot noise and conductance fluctuations, respectively. An appendix contains a calculation whose result is central to the phase-space approach employed in Sec. III.

\section{PRELIMINARIES}

We follow the usual approach to obtain semiclassical approximations to transmission by employing the Landauer formalism ${ }^{2,3}$ and introducing semiclassical representations for Green's functions. In the absence of spin-orbit interactions, this procedure is well established. ${ }^{25-27}$ Here, we briefly describe the extensions required by the presence of spin-orbit interactions (see also Ref. 20).

\section{A. Landauer formalism with spin}

The Landauer formalism provides a link between conductance coefficients, as defined through

$$
I_{n}=\sum_{m} g_{n m} V_{m}
$$

and $S$-matrix elements. In Eq. (2), the indices label the leads, $V_{m}$ is the voltage applied at lead $m$, and $I_{n}$ is the current through lead $n$. Here, the number of leads may be arbitrary. An $S$-matrix element $S_{\alpha_{n} \alpha_{m}^{\prime}}^{n m}$ is defined as the transition amplitude between an asymptotic incoming state in the lead $m$, characterized by the collection $\alpha_{m}^{\prime}$ of its quantum numbers, to an asymptotic outgoing state in the lead $n$, accordingly characterized by $\alpha_{n}$.

In Refs. 26 and 27, the Landauer formalism was derived from the Schrödinger equation in linear response theory, making use of an appropriate Kubo-Greenwood formula. We first remark that an inclusion of spin, interacting with the translational degrees of freedom via a Zeeman, Rashba, or Dresselhaus coupling, into this method causes no problems. Although the current density is modified, its conservation in the form required for the Kubo-Greenwood expression of the conductivity to hold is indeed guaranteed. One then obtains for transmission (i.e., $m \neq n$ )

$$
g_{n m}=-\frac{e^{2}}{h} \int_{0}^{\infty} d E f_{\beta}^{\prime}(E) \sum_{\alpha_{n}, \alpha_{m}^{\prime}}\left|S_{\alpha_{n} \alpha_{m}^{\prime}}^{n m}\right|^{2},
$$

and for reflection (i.e., $m=n$ )

$$
g_{n n}=\frac{e^{2}}{h} \int_{0}^{\infty} d E f_{\beta}^{\prime}(E)\left[(2 s+1) N_{n}-\sum_{\alpha_{n}, \alpha_{n}^{\prime}}\left|S_{\alpha_{n} \alpha_{n}^{\prime}}^{n n}\right|^{2}\right] .
$$

Here, $N_{n}$ is the number of open channels in the lead $n$ (without spin degeneracy) at energy $E$, and $f_{\beta}(E)$ denotes the Fermi distribution function at inverse temperature $\beta$. Of course, this requires the spin quantum number $s$ to be halfinteger.

In the next step, $S$-matrix elements have to be related to Green's functions $G\left(\mathbf{x}, \mathbf{x}^{\prime}, E\right)$. These satisfy the following equations:

$$
\left\{\frac{1}{2 m}\left[\hat{\mathbf{p}}-\frac{e}{c} \mathbf{A}(\hat{\mathbf{x}})\right]^{2}+\hat{\mathbf{s}} \cdot \mathbf{C}(\hat{\mathbf{x}}, \hat{\mathbf{p}})-E\right\} G\left(\mathbf{x}, \mathbf{x}^{\prime}, E\right)=\delta\left(\mathbf{x}-\mathbf{x}^{\prime}\right)
$$

and

$$
\begin{aligned}
\left\{\frac{1}{2 m}\left[\hat{\mathbf{p}}^{\prime}+\frac{e}{c} \mathbf{A}\left(\hat{\mathbf{x}}^{\prime}\right)\right]^{2}-E\right\} G\left(\mathbf{x}, \mathbf{x}^{\prime}, E\right) \\
+\mathbf{C}^{*}\left(\hat{\mathbf{x}}^{\prime}, \hat{\mathbf{p}}^{\prime}\right) G\left(\mathbf{x}, \mathbf{x}^{\prime}, E\right) \hat{\mathbf{s}}=\delta\left(\mathbf{x}-\mathbf{x}^{\prime}\right) .
\end{aligned}
$$

The unusual form of the second equation is dictated by the fact that $G\left(\mathbf{x}, \mathbf{x}^{\prime}, E\right)$ is a Hermitian $(2 s+1) \times(2 s+1)$ matrix in spin space. In the following, we will always choose advanced Green's functions, fully characterized by Eqs. (5) and (6) as well as the condition that, asymptotically in the leads, they contain only outgoing contributions.

As in the case without $\operatorname{spin}^{26}$ one can express the $S$-matrix elements in terms of the (advanced) Green's function. To this end, one replaces the disorder potential $U(\mathbf{x})$ occurring in Ref. 26 with the spin-orbit interaction term. Due to the Hermiticity of the coupling, one can then proceed as in Ref. 26. Up to a global phase factor, for $m \neq n$ this yields

$$
\begin{aligned}
S_{\alpha_{n} \alpha_{m}^{\prime}}^{n m}= & \frac{2 \hbar^{2}}{i m} \sqrt{\frac{k_{a_{n}} k_{a_{m}^{\prime}}}{W_{m} W_{n}}} \int_{0}^{W_{n}} d y_{n} \int_{0}^{W_{m}} d y_{m}^{\prime} \sin \left(\frac{a_{n} \pi y_{n}}{W_{n}}\right) \\
& \times \sin \left(\frac{a_{m}^{\prime} \pi y_{m}^{\prime}}{W_{m}}\right) G_{\sigma \sigma^{\prime}}\left(\mathbf{x}_{n}, \mathbf{x}_{m}^{\prime}, E\right),
\end{aligned}
$$

and for $m=n$

$$
\begin{aligned}
S_{\alpha_{n} \alpha_{n}^{\prime}}^{n n}= & \frac{2 \hbar^{2}}{i m} \frac{\sqrt{k_{a_{n}} k_{a_{n}^{\prime}}}}{W_{n}} \int_{0}^{W_{n}} d y_{n} \int_{0}^{W_{n}} d y_{n}^{\prime} \sin \left(\frac{a_{n} \pi y_{n}}{W_{n}}\right) \\
& \times \sin \left(\frac{a_{n}^{\prime} \pi y_{n}^{\prime}}{W_{n}}\right) G_{\sigma \sigma^{\prime}}\left(\mathbf{x}_{n}, \mathbf{x}_{n}^{\prime}, E\right)+\delta_{\alpha_{n} \alpha_{n}^{\prime} .}
\end{aligned}
$$

Here, we have introduced coordinates $\mathbf{x}_{n}=\left(x_{n}, y_{n}\right)$, where $x_{n}$ $\geqslant 0$ is a longitudinal, outward running coordinate in the lead $n$ and $0 \leqslant y_{n} \leqslant W_{n}$ is the corresponding transversal coordinate (see also Fig. 1). The transversal quantum number is $a_{n}$ $=1, \ldots, N_{n}$ with associated wave number $k_{a_{n}}$ $=\sqrt{2 m E / \hbar^{2}-a_{n}^{2} \pi^{2} / W_{n}^{2}}$. The number $N_{n}$ of open transversal channels then is the largest integer $a_{n}$ that leaves the wave number real. Moreover, $\sigma=-s, \ldots, s$ is a spin index such that altogether $\alpha_{n}=\left(E, a_{n}, \sigma\right)$. 

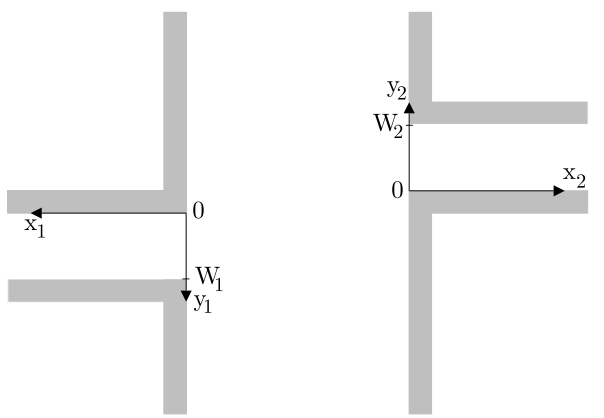

FIG. 1. Sketch of the geometry.

We remark that in Eqs. (7) and (8) the points $\mathbf{x}_{n}, \mathbf{x}_{m}^{\prime}$ can be chosen anywhere in the respective leads. For later convenience, we take them on the connection of the leads to the cavity, i.e., with $x_{n}=0=x_{m}^{\prime}$.

\section{B. Semiclassical Green's function and transmission amplitudes}

In order to proceed further, one requires a semiclassical representation for the Green's function defined in Eqs. (5) and (6). In Ref. 21, this was achieved through an asymptotic expansion in powers of Planck's constant $\hbar$ for the quantum propagator generated by the Hamiltonian (1) which yielded, after a Fourier transformation, a respective semiclassical expansion for the Green's function. The range of validity of this procedure follows from the observation that since the spin operator $\hat{\mathbf{s}}$ is linear in $\hbar$, the energy scale of the spinorbit interaction term becomes small as compared to the kinetic term in the limit $\hbar \rightarrow 0$. This condition is equivalent to the spin-precession length being large compared to the Fermi wavelength. In semiconductor heterostructures, this requirement is usually fulfilled.

The semiclassical representation for the Green's function obtained in Ref. 21 reads

$$
G\left(\mathbf{x}, \mathbf{x}^{\prime}, E\right) \sim \sum_{\gamma\left(\mathbf{x}, \mathbf{x}^{\prime}\right)} A_{\gamma}\left(\mathbf{x}, \mathbf{x}^{\prime}, E\right) \exp \left((i / \hbar) S_{\gamma}\left(\mathbf{x}, \mathbf{x}^{\prime}, E\right)\right)
$$

as $\hbar \rightarrow 0$. The sum extends over all classical trajectories $\gamma\left(\mathbf{x}, \mathbf{x}^{\prime}\right)$ generated by the classical Hamiltonian

$$
H_{0}(\mathbf{x}, \mathbf{p})=\frac{1}{2 m}\left[\mathbf{p}-\frac{e}{c} \mathbf{A}(\mathbf{x})\right]^{2}
$$

(plus reflections from hard walls) that run from $\mathbf{x}^{\prime}$ to $\mathbf{x}$ at energy E. Choosing $\left(\mathbf{x}, \mathbf{x}^{\prime}\right)=\left(\mathbf{x}_{n}, \mathbf{x}_{m}^{\prime}\right)$ as in Eqs. (7) and (8), the relevant trajectories are those that enter the cavity at lead $m$ and leave through lead $n$. Moreover, $S_{\gamma}\left(\mathbf{x}, \mathbf{x}^{\prime}, E\right)$ is the classical action of the trajectory, and the leading order of the amplitude $A_{\gamma}\left(\mathbf{x}, \mathbf{x}^{\prime}, E\right)$ reads

$$
A_{\gamma}\left(\mathbf{x}, \mathbf{x}^{\prime}, E\right)=\frac{e^{-i(\pi / 2) \nu_{\gamma}}}{i \hbar \sqrt{2 \pi i \hbar}} \sqrt{C_{\gamma}} D_{\gamma}\left(\mathbf{x}^{\prime}, \mathbf{p}^{\prime}, t\right)[1+O(\hbar)] .
$$

Here, $\nu_{\gamma}$ is a Maslov index of the trajectory $\gamma$, and

$$
C_{\gamma}:=\left|\operatorname{det}\left(\begin{array}{cc}
\frac{\partial^{2} S_{\gamma}}{\partial \mathbf{x} \partial \mathbf{x}^{\prime}} & \frac{\partial^{2} S_{\gamma}}{\partial \mathbf{x} \partial E} \\
\frac{\partial^{2} S_{\gamma}}{\partial \mathbf{x}^{\prime} \partial E} & \frac{\partial^{2} S_{\gamma}}{\partial E^{2}}
\end{array}\right)\right| .
$$

The contribution of the spin is, in leading semiclassical order, completely contained in the spin-transport matrix $D_{\gamma}\left(\mathbf{x}^{\prime}, \mathbf{p}^{\prime}, t\right)$. This is the spin-s representation of the spin propagator $d_{\gamma}\left(\mathbf{x}^{\prime}, \mathbf{p}^{\prime}, t\right)$, which is defined as a solution of the equation

$$
\frac{d}{d t} d_{\gamma}\left(\mathbf{x}^{\prime}, \mathbf{p}^{\prime}, t\right)+\frac{i}{2} \mathbf{C}(\mathbf{X}(t), \mathbf{P}(t)) \cdot \boldsymbol{\sigma} d_{\gamma}\left(\mathbf{x}^{\prime}, \mathbf{p}^{\prime}, t\right)=0,
$$

with initial condition $d_{\gamma}\left(\mathbf{x}^{\prime}, \mathbf{p}^{\prime}, 0\right)=1$. Here, $(\mathbf{X}(t), \mathbf{P}(t))$ is the point in phase space of the classical trajectory $\gamma$ at time $t$. Its initial point at time $t=0$ is $\left(\mathbf{x}^{\prime}, \mathbf{p}^{\prime}\right)$. Moreover, $\boldsymbol{\sigma}$ is the vector of Pauli spin matrices. Therefore, $d_{\gamma}$ is an SU(2) matrix that can be seen as a propagator for the spin along the classical trajectory $\gamma$.

Upon dividing the trajectory $\gamma$ into two pieces $\gamma_{1}$ and $\gamma_{2}$, such that $t=t_{1}+t_{2}$, the spin propagator is clearly multiplicative. Since $D_{\gamma}$ arises from a group representation, it inherits this multiplicative property from the propagator, i.e.,

$$
D_{\gamma}\left(\mathbf{x}^{\prime}, \mathbf{p}^{\prime}, t_{1}+t_{2}\right)=D_{\gamma_{2}}\left(\mathbf{X}\left(t_{1}\right), \mathbf{P}\left(t_{1}\right), t_{2}\right) D_{\gamma_{1}}\left(\mathbf{x}^{\prime}, \mathbf{p}^{\prime}, t_{1}\right) .
$$

This relation will be used extensively in Sec. III.

In order to obtain a semiclassical representation of transmission amplitudes, we insert expression (9) into Eq. (7). Then, the integrals over $y$ and $y^{\prime}$, respectively, are evaluated, asymptotically as $\hbar \rightarrow 0$, with the method of stationary phase. In this context, we stress the following important observation: The number of accessible transversal states (including spin) in the $n$th lead is $(2 s+1) N_{n}=(2 s+1)\left[\sqrt{2 m E} W_{n} /(\pi \hbar)\right]$, where $[x]$ denotes the integer part of $x \in \mathbb{R}$. We choose the widths $W_{n}$ of the leads to formally shrink proportionally to $\hbar$ in this limit (compare also Ref. 15) and hence set $W_{n}=\widetilde{W}_{n} \hbar$, to the effect that the sine factors in Eqs. (7) and (8) contribute rapidly oscillating phases. These have to be taken into account when determining stationary points of the total phases in the integrals. The condition of stationary phase hence imposes the following restrictions on the transversal momenta:

$$
p_{y}^{\prime}=-\frac{\partial S_{\gamma}}{\partial y_{m}^{\prime}}= \pm \frac{a_{m}^{\prime} \pi}{\widetilde{W}_{m}}
$$

and

$$
p_{y}=\frac{\partial S_{\gamma}}{\partial y_{n}}=\mp \frac{a_{n} \pi}{\widetilde{W}_{n}},
$$

upon entry and exit, respectively, of the trajectories. If the points of entry and exit are free of magnetic fields, and thus $\mathbf{p}=m \dot{\mathbf{x}}$ at these points, one can characterize the trajectories in terms of the angles $\theta$ and $\theta^{\prime}$, under which they enter and leave the cavity with respect to the longitudinal directions of the leads (see also Fig. 2). These angles are related to the 
transversal momenta [Eqs. (15) and (16)] through $\sin \theta$ $=p_{y} / \sqrt{2 m E}$ and $\sin \theta^{\prime}=p_{y}^{\prime} / \sqrt{2 m E}$. If one wished to keep the widths of the openings fixed, however, the method of stationary phase would enforce the conditions $p_{y}^{\prime}=0=p_{y}$ upon the trajectories, thus leading to semiclassical expressions different from the ones we use henceforth. In order to keep conditions equivalent to Eqs. (15) and (16), one then would have to consider large transversal quantum numbers. This could be achieved by introducing rescaled quantum numbers $\widetilde{a}_{n}=a_{n} \hbar$, kept at a fixed value. In that context, the limit of large numbers $N_{n}$ of open transversal channels is required (see also Ref. 16).

Collecting now all terms that emerge in the stationary phase calculation finally leads to the following leading semiclassical contribution to the $S$-matrix elements:

$$
S_{\alpha_{n} \alpha_{m}^{\prime}}^{n m} \sim \sum_{\gamma\left(\theta, \theta^{\prime}\right)} B_{\gamma\left(\theta, \theta^{\prime}\right)} D_{\gamma\left(\theta, \theta^{\prime}\right)}^{\sigma \sigma^{\prime}} \exp \left((i / \hbar) S_{\gamma\left(\theta, \theta^{\prime}\right)}\right),
$$

where the sum extends over all trajectories that run from lead $m$ through the cavity to lead $n$ and are characterized by conditions (15) and (16), expressed in terms of the angles of entry and exit. The explicit form of the factor $B_{\gamma\left(\theta, \theta^{\prime}\right)}$ is the same as if there were no spin present, ${ }^{26}$

$$
\begin{aligned}
B_{\gamma\left(\theta, \theta^{\prime}\right)}= & \sqrt{\frac{i \pi \hbar}{2 W_{m} W_{n}}} \frac{\operatorname{sgn}\left( \pm a_{m}^{\prime}\right) \operatorname{sgn}\left( \pm a_{n}\right)}{\left|\cos \theta \cos \theta^{\prime} M_{\gamma\left(\theta, \theta^{\prime}\right)}^{21}\right|^{1 / 2}} \exp \left(i \pi \left(\frac{ \pm a_{m}^{\prime} y_{m}^{\prime}}{W_{m}}\right.\right. \\
& \left.\left.+\frac{ \pm a_{n} y_{n}}{W_{n}}-\frac{1}{2} \mu_{\gamma\left(\theta, \theta^{\prime}\right)}\right)\right) .
\end{aligned}
$$

Here, $M_{\gamma\left(\theta, \theta^{\prime}\right)}^{21}$ is an element of the monodromy matrix of $\gamma\left(\theta, \theta^{\prime}\right)$ that arises from the matrix appearing in Eq. (12) by a restriction to the phase-space directions transversal to the trajectory. Furthermore, $\mu_{\gamma\left(\theta, \theta^{\prime}\right)}$ is a modified Maslov index that contains the index $\nu_{\gamma\left(\theta, \theta^{\prime}\right)}$ from Eq. (11) and additional phases resulting from the stationary phase calculation of the integrals over $y_{n}$ and $y_{m}^{\prime}$.

The above result [Eq. (17)] primarily refers to transmission amplitudes $(n \neq m)$, but can be carried over to the case of reflection $(n=m)$. The reason for this is that the additional term $\delta_{\alpha_{n} \alpha_{n}^{\prime}}$ in Eq. (8) is canceled by the contribution of direct trajectories in the opening of the lead $n$ that never enter the cavity. ${ }^{4}$

The ultimate goal being a semiclassical calculation of the conductance coefficients [Eqs. (3) and (4)], one therefore requires the evaluation of double sums,

$$
\left|S_{\alpha_{n} \alpha_{m}^{\prime}}^{n m}\right|^{2} \sim \sum_{\gamma\left(\theta, \theta^{\prime}\right)} \sum_{\gamma^{\prime}\left(\theta, \theta^{\prime}\right)} B_{\gamma} B_{\gamma^{\prime}}^{*} D_{\gamma}^{\sigma \sigma^{\prime}} D_{\gamma^{\prime}}^{\sigma \sigma^{\prime *}} \exp \left((i / \hbar)\left(S_{\gamma}-S_{\gamma^{\prime}}\right)\right) \text {, }
$$

over classical trajectories. This will be the task for the rest of this paper.

To simplify the calculations, from now on we restrict our attention to the case of two leads. With an incoming wave in the lead $m=1$, we are thus dealing with the transmission coefficient $g_{21}$ and the reflection coefficient $g_{11}$. To this end, we will determine the transmission matrix $S^{21}$ and the reflec- tion matrix $S^{11}$, leading to the transmission and reflection coefficients

$$
\mathcal{T}=\left.\sum_{\alpha_{2}, \alpha_{1}^{\prime}}\left|S_{\alpha_{2} \alpha_{1}^{\prime}}^{21}, \quad \mathcal{R}=\sum_{\alpha_{2}, \alpha_{2}^{\prime}}\right| S_{\alpha_{2} \alpha_{2}^{\prime}}^{22}\right|^{2},
$$

respectively. Hence, at zero temperature the current through lead 2 is

$$
I_{2}=\frac{e^{2}}{h}\left\{\mathcal{T} V_{1}+\left[\mathcal{R}-(2 s+1) N_{2}\right] V_{2}\right\},
$$

where $\mathcal{T}$ and $\mathcal{R}$ are taken at the Fermi energy $E_{F}$. Together with the condition $g_{21}+g_{22}=0$, expressing that equal voltages at both leads produce no current, this yields the relation

$$
I_{2}=\frac{e^{2}}{h} \mathcal{T}\left(V_{1}-V_{2}\right) \text {. }
$$

\section{SEMICLASSICAL CALCULATION OF CONDUCTIVITY COEFFICIENTS}

The calculation of the double sum [Eq. (19)] over classical trajectories requires input from dynamical properties of the associated classical system. With spin-orbit interactions present, one therefore first has to identify an appropriate classical system. Moreover, ergodic properties of the classical system imply necessary ingredients for the further calculation. The diagonal contribution to the double sum is evaluated with a sum rule,,${ }^{9,20}$ whereas the nondiagonal terms are evaluated following the Sieber-Richter method. . $^{5,15,20}$

\section{A. Classical spin-orbit dynamics}

The classical dynamics that enters the semiclassical representation (9) consists of two parts: ${ }^{21}$ the motion of the point particle generated by the Hamiltonian (10), including elastic reflections from hard walls, and the spin that is driven by this motion according to Eq. (13). These contributions can be combined into a single dynamics on a spin-orbit phase space. ${ }^{22}$ The relevant classical trajectory is $(\mathbf{X}(t), \mathbf{P}(t), g(t))$, with initial condition $\left(\mathbf{x}^{\prime}, \mathbf{p}^{\prime}, g^{\prime}\right)$ at $t=0$. Here, $g \in \mathrm{SU}(2)$ and $g(t)=d_{\gamma}\left(\mathbf{x}^{\prime}, \mathbf{p}^{\prime}, t\right) g$ provides the spin part of the combined motion. We remark that this description of spin appears quantum mechanical. However, by passing to expectation values of the spin operator $d_{\gamma 2}^{\dagger} \frac{1}{2} \boldsymbol{\sigma} d_{\gamma}$ in normalized spin states $\chi$ (Heisenberg picture), the spin variable becomes a unit vector $\left\langle\chi, d_{\gamma 2}^{\dagger} \boldsymbol{\sigma} d_{\gamma} \chi\right\rangle$. Hence, the spin part of the combined phase space is a unit sphere. The two views of the spin motion, either on $\mathrm{SU}(2)$ or on a unit sphere, are, in fact, equivalent. ${ }^{21}$ In both cases, we will therefore speak of classical spin-orbit dynamics.

Ergodicity is a concept developed for closed systems. It can, however, be suitably extended to open systems of the kind under consideration here. To this end, one divides the configuration space $Q$ of the device into a closed part $Q_{c}$, consisting of the cavity with the leads truncated and the openings closed, plus the infinite leads. From now on, we suppose the shape of the closed part to form a chaotic billiard, ensuring ergodicity of the motion inside the cavity. 
Then, $\rho(t)$ is the probability for a typical trajectory to stay within the cavity at least up to time $t$. For large times,

$$
\rho(t) \sim \exp (-t / \tau), \quad t \rightarrow \infty,
$$

with inverse dwell time

$$
\frac{1}{\tau}=\frac{\hbar}{m A}\left(N_{1}+N_{2}\right),
$$

in which $A$ denotes the area of the closed part $Q_{c}$. For the associated part of phase space, we also introduce the volume

$$
\Sigma(E)=\int_{Q_{c}} d^{2} x \int_{\mathbb{R}^{2}} d^{2} p \delta\left(E-H_{0}(\mathbf{x}, \mathbf{p})\right)=2 \pi m A
$$

of the energy shell. This expression has no integration over the spin part, since the Hamiltonian is independent thereof, and an integration over $\mathrm{SU}(2)$ with respect to Haar measure $d g$ yields 1 .

For the open system, the concept of ergodicity has to be modified in that the possibility of a trajectory to leave the cavity must be taken into account. When the motion inside the cavity is ergodic, this leads to the following relation between phase-space averages and time averages over typical spin-orbit trajectories:

$$
\begin{aligned}
& \left\langle\int_{0}^{T} d t f(\mathbf{X}(t), \mathbf{P}(t), g(t))\right\rangle \\
& \sim \frac{1}{\Sigma(E)} \int_{0}^{T} d t \rho(t) \int_{Q_{c}} d^{2} x \int_{\mathrm{R}^{2}} d^{2} p \int_{\mathrm{SU}(2)} d g f(\mathbf{x}, \mathbf{p}, g) \\
& \quad \times \delta\left(E-H_{0}(\mathbf{x}, \mathbf{p})\right)
\end{aligned}
$$

as $T \rightarrow \infty$. Here, $f$ is an arbitrary function on the combined phase space and $\langle\cdots\rangle$ denotes an average over initial conditions. This relation, which properly reflects the chaotic nature of the combined classical spin-orbit motion, provides the basis for further use of dynamical properties in the calculation of the sum [Eq. (19)] over classical trajectories.

The stronger mixing property, which we also assume to hold henceforth, means that correlations of two spin-orbit observables $f$ and $h$ decay, i.e.,

$$
\begin{aligned}
& \lim _{t \rightarrow \infty} \int_{Q_{c}} d^{2} x \int_{\mathbb{R}^{2}} d^{2} p \int_{\mathrm{SU}(2)} d g h(\mathbf{X}(t), \mathbf{P}(t), g(t)) f(\mathbf{x}, \mathbf{p}, g) \delta\left(E-H_{0}(\mathbf{x}, \mathbf{p})\right) \\
& \quad=\frac{1}{\sum(E)} \int_{Q_{c}} d^{2} x \int_{\mathbb{R}^{2}} d^{2} p \int_{\mathrm{SU}(2)} d g h(\mathbf{x}, \mathbf{p}, g) \delta\left(E-H_{0}(\mathbf{x}, \mathbf{p})\right) \int_{Q_{c}} d^{2} x^{\prime} \int_{\mathbb{R}^{2}} d^{2} p^{\prime} \int_{\mathrm{SU}(2)} d g^{\prime} f\left(\mathbf{x}^{\prime}, \mathbf{p}^{\prime}, g^{\prime}\right) \delta\left(E-H_{0}\left(\mathbf{x}^{\prime}, \mathbf{p}^{\prime}\right)\right) .
\end{aligned}
$$

We stress that ergodicity and mixing of the spin-orbit dynamics do not simply follow from chaotic properties of the underlying translational motion. They require, in addition, that the spin-orbit coupling is sufficiently complex in order to exclude trivial spin motions.

\section{B. Transmission and reflection coefficients in the configuration-space approach}

In the first step, we calculate the leading semiclassical contribution to transmission and reflection coefficients from Eq. (19), averaged over a small energy window, by using the configuration-space approach. Such a calculation has been performed previously, ${ }^{20}$ however, with a sum rule that only takes the particle motion into account. The spin contribution was built in subsequently, assuming that traces of products of spin-transport matrices can be replaced by certain averages. Here, we reproduce the result obtained in Ref. 20 by using a sum rule for the complete spin-orbit dynamics that follows from Eq. (26). Thus, we base the assumptions made in Ref. 20 on a firm dynamical ground.

As $\hbar \rightarrow 0$, the terms in the double sum [Eq. (19)] are highly oscillatory, except for contributions with $S_{\gamma}=S_{\gamma^{\prime}}$. Generically, if no symmetries are present, this only occurs for the diagonal $\gamma^{\prime}=\gamma$. In the event that time-reversal invariance is not broken, however, the time-reversed trajectory $\gamma^{-1}$ has the same action as $\gamma$. Of course, $\gamma^{-1}$ is only among the trajectories to be summed over in the case of reflection $(n=1$ $=m$ ) when, moreover, $\theta=\theta^{\prime}$, i.e., only for $S_{\alpha_{1} \alpha_{1}^{\prime}}^{11}$ with $a_{1}=a_{1}^{\prime}$. All further terms are oscillatory, with a decreasing importance of their contribution, after averaging over an energy window, when the action differences increase. Below, we calculate the two leading contributions to the quantity

$$
\sum_{\sigma, \sigma^{\prime}=-s}^{s}\left|S_{\alpha_{n} \alpha_{m}^{\prime}}^{n m}\right|^{2} \sim \sum_{\gamma, \gamma^{\prime}} B_{\gamma} B_{\gamma^{\prime}}^{*} \operatorname{Tr}\left(D_{\gamma} D_{\gamma^{\prime}}^{\dagger}\right) \exp \left((i / \hbar)\left(S_{\gamma}-S_{\gamma^{\prime}}\right)\right)
$$

for systems with time-reversal invariance: (i) the diagonal contribution in which the sum over $\gamma^{\prime}$ is restricted to $\gamma^{\prime}=\gamma$ (for transmission) or $\gamma^{\prime}=\gamma^{ \pm 1}$ (for reflection) and (ii) the oneloop contribution in which the sums over $\gamma$ and $\gamma^{\prime}$ are confined to so-called Sieber-Richter pairs (see also Ref. 20).

Due to the unitarity of the spin-transport matrices, in the diagonal case, terms with $\gamma^{\prime}=\gamma$ yield a spin contribution of $\operatorname{Tr}\left(D_{\gamma} D_{\gamma}^{\dagger}\right)=2 s+1$. Thus, the diagonal contribution to Eq. (28) 
can immediately be obtained from the respective result without spin, 4,9

$$
\left\langle\sum_{\sigma, \sigma^{\prime}=-s}^{s}\left|S_{\alpha_{n} \alpha_{m}^{\prime}}^{n m}\right|_{\text {diag }}^{2}\right\rangle_{\Delta E} \sim \frac{2 s+1}{N_{1}+N_{2}}
$$

In the case of reflection $(n=1=m)$ with $a_{1}=a_{1}^{\prime}$, an additional diagonal contribution arises from the terms with $\gamma^{\prime}=\gamma^{-1}$, if time-reversal invariance is unbroken. Its spin contribution is $\operatorname{Tr}\left(D_{\gamma} D_{\gamma^{-1}}^{\dagger}\right)=\operatorname{Tr}\left(D_{\gamma}^{2}\right)$. One hence requires a suitable sum rule that incorporates the combined classical spin-orbit motion. For this purpose, we choose the function

$$
\begin{aligned}
f(\mathbf{X}(t), \mathbf{P}(t), g(t))= & \frac{1}{m} \delta(\vartheta(t)-\theta) \delta(x(t))[\Theta(y(t))-\Theta(y(t) \\
& \left.\left.-W_{1}\right)\right] \operatorname{Tr}\left(\pi_{s}\left[g(t) g(0)^{-1}\right]\right)^{2}
\end{aligned}
$$

in Eq. (26). Here, $\pi_{s}(g)$ denotes the spin-s representation of $g \in \mathrm{SU}(2), \vartheta$ is the angular variable in planar polar coordinates for $\mathbf{p}$, and $\Theta(y)$ is a Heavyside step function. An evaluation of Eq. (26) with the function in Eq. (30) then leads to the sum rule $($ as $T \rightarrow \infty)$

$$
\sum_{\gamma, T_{\gamma} \leqslant T}\left|B_{\gamma}\right|^{2} \operatorname{Tr}\left(D_{\gamma}^{2}\right) \sim \frac{\pi}{2 \tilde{W}_{1}} \frac{(-1)^{2 s}}{2 \pi m A} \int_{0}^{T} d t \rho(t) .
$$

After an average over a small window in energy this, together with Eq. (29), finally yields the semiclassical result

$$
\left\langle\sum_{\sigma, \sigma^{\prime}=-s}^{s}\left|S_{\alpha_{1} \alpha_{1}^{\prime}}^{11}\right|_{\text {diag }}^{2}\right\rangle_{\Delta E} \sim \frac{2 s+1+(-1)^{2 s} \delta_{a_{1} a_{1}^{\prime}}}{N_{1}+N_{2}}
$$

for the diagonal contribution to Eq. (28). For $s=1 / 2$, the right-hand side is $1 /\left(N_{1}+N_{2}\right)$.

Sieber-Richter pairs of trajectories are characterized by the fact that one trajectory possesses a self-crossing with a small crossing angle $\varepsilon$, thus forming a loop. The partner trajectory then looks like the former one cut open at the self-crossing, but with the loop direction reversed and then glued together, such that the self-crossing is replaced by an almost-crossing (see Fig. 2). In principle, the trajectories in such pairs can have an arbitrary number of self-crossings, but the magnitude of their contributions to Eq. (28) decreases with increasing action differences. These, in turn, grow with the number of places in which a self-crossing of one trajectory is paired with an almost-crossing of the corresponding partner trajectory. The most important ("one-loop") contribution comes from pairs which differ in one crossing. In order to calculate the one-loop contribution, one requires the distribution of the crossing angles $\varepsilon$ for pairs of trajectories with loops of duration $T$,

$$
P_{S}(\varepsilon, T)=\frac{1}{\Sigma(E)} \int_{Q_{c}} d^{2} x^{\prime} \int_{\mathbb{R}^{2}} d^{2} p^{\prime} \int_{T_{\min }(\varepsilon)}^{T} d t_{l} p_{S}\left(\varepsilon, T, t_{l}\right) .
$$

Here, $p_{S}\left(\varepsilon, T, t_{l}\right)$ is a density of crossing angles defined as

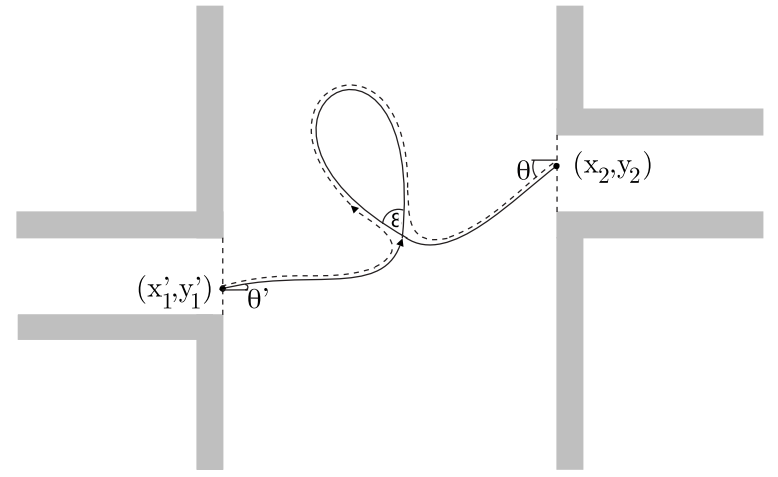

FIG. 2. A Sieber-Richter pair of trajectories.

$$
\begin{aligned}
p_{S}\left(\varepsilon, T, t_{l}\right)= & \int_{0}^{T-t_{l}} d t_{s}|J| \delta\left(E-H_{0}\left(\mathbf{P}\left(t_{s}\right)\right)\right) \operatorname{Tr}\left(\pi_{s}\{g(t)\right. \\
& \left.\left.\times[g(0)]^{-1}\right\}\right)^{2} \delta\left(\varepsilon-\kappa\left(t_{s}, t_{l}\right)\right) \delta\left(\mathbf{X}\left(t_{s}\right)-\mathbf{X}\left(t_{s}+t_{l}\right)\right),
\end{aligned}
$$

where $\kappa\left(t_{s}, t_{l}\right)$ denotes the angle between the velocities $\mathbf{v}\left(t_{s}\right)$ and $\mathbf{v}\left(t_{s}+t_{l}\right)$. Given a crossing angle $\varepsilon$, the minimal duration for a loop to close is $T_{\min }(\varepsilon)$. In chaotic systems, this quantity behaves like $T_{\min }(\varepsilon)=O(\log \varepsilon)$ as $\varepsilon \rightarrow 0 .{ }^{5}$ Furthermore,

$$
|J|=\left|\mathbf{v}\left(t_{s}\right) \times \mathbf{v}\left(t_{s}+t_{l}\right)\right|=\left|\mathbf{v}\left(t_{s}\right)\right|\left|\mathbf{v}\left(t_{s}+t_{l}\right)\right| \sin \kappa\left(t_{s}, t_{l}\right)
$$

is a Jacobian, and $t_{s}, t_{l}$ denote the time along the trajectory up to the starting point of the loop and along the loop, respectively.

Assuming that the classical spin-orbit dynamics is not only ergodic, but also mixing, the distribution in Eq. (33) can be calculated further. It can be identified as the left-hand side of an appropriate relation of the type of Eq. (27). The righthand side then yields, as $\varepsilon \rightarrow 0$,

$$
P_{S}(\varepsilon, T) \sim \frac{(-1)^{2 s}}{\pi A} \frac{2 E}{m} \sin \varepsilon\left[\frac{T^{2}}{2}-T T_{\min }(\varepsilon)+\frac{T_{\text {min }}^{2}(\varepsilon)}{2}\right] .
$$

This expression differs from the respective one without spin that was obtained in Ref. 5 only by a factor $(-1)^{2 s}$, i.e., a sign in the case $s=1 / 2$. With this information at hand, the oneloop contribution can be calculated as in the case without spin, ${ }^{9}$ finally yielding

$$
\left\langle\sum_{\sigma, \sigma^{\prime}=-s}^{s}\left|S_{\alpha_{1} \alpha_{2}^{\prime}}^{21}\right|_{1-\text { loop }}^{2}\right\rangle_{\Delta E} \sim-\frac{(-1)^{2 s}}{\left(N_{1}+N_{2}\right)^{2}} .
$$

This is in accordance with what has been obtained in Ref. 20 .

\section{Transmission coefficients in the phase-space approach}

Higher orders in the "loop expansion" described above have been calculated previously for spectral form factors ${ }^{7}$ as well as for conductance coefficients for systems without spin contributions. ${ }^{10}$ The approach taken in these papers utilizes trajectories in classical phase space and identifies the pairs of self-crossings and/or almost-crossings in configuration space 
as pairs of trajectories with almost-crossings in phase space, which differ in the way they are connected at the (almost) crossings. This point of view opens the possibility for a classification of the trajectory pairs in terms of their encounters. ${ }^{7}$ Here, we follow this phase-space approach and amend the previous result ${ }^{10}$ with the contribution of the spin-orbit coupling.

To be more precise, we consider trajectories that possess close self-encounters (in phase space), in which two or more short stretches of the trajectory are almost identical, possibly up to time reversal. These stretches are connected by long parts of the trajectory, which we call loops. We then form pairs $\left(\gamma, \gamma^{\prime}\right)$ of such trajectories in which $\gamma$ and $\gamma^{\prime}$ are almost identical (up to time reversal) along the loops, but differ from each other in the way the loops are connected in the encounter region. In order to quantify these encounters, we introduce a vector $\vec{v}$, whose $l$ th component, $v_{l}$, denotes the number of encounters with $l$ stretches. Hence, the total number of encounters is $V=\Sigma_{l \geqslant 2} v_{l}$, with a total of $L=\Sigma_{l \geqslant 2} l v_{l}$ stretches involved. In general, however, given a vector $\vec{v}$, there will be $N(\vec{v}) \geqslant 1$ different trajectory pairs associated with it. These may, e.g., differ in the order the loops connect the encounters, or in the relative directions, in which the encounter stretches are traversed.

To reveal the phase-space structure of trajectory pairs and to compute their contributions to Eq. (19), one introduces Poincaré sections, which cut the trajectories into pieces. In order to adapt this cutting to the sequence of encounters and loops, one chooses a Poincaré section in every of the $V$ given encounter regions. We then denote by $t_{\alpha, j}^{\prime}, j=1, \ldots, l_{\alpha}, \alpha$ $=1, \ldots, V$ the times at which the encounter stretches pierce this section, and by $t_{e n c}^{\alpha}$, the duration of the encounters. To this cutting of the trajectories corresponds the splitting

$$
D_{\gamma}=D_{L+1} D_{L} \cdots D_{1}
$$

of the spin-transport matrices which, with an obvious notation, follows from the composition rule [Eq. (14)]. The spin transport along the partner trajectory then reads

$$
D_{\gamma^{\prime}} \approx D_{L+1} D_{k_{L}}^{\eta_{L}} \cdots D_{k_{2}}^{\eta_{2}} D_{1} .
$$

Here, $\eta_{j}= \pm 1$, depending on the relative orientation of the trajectory between the $(j-1)$ st and the $j$ th cutting of $\gamma$ and $\gamma^{\prime}$, respectively, through the Poincare section. We notice that at this point, time-reversal invariance enters crucially. Moreover, the indices $k_{j}$ take care of the fact that in $\gamma$ and $\gamma^{\prime}$, the loops may be traversed in different successions. Thus, the spin-dependent weights in Eq. (28) for each pair of trajectories are approximately given by

$$
\operatorname{Tr}\left(D_{\gamma} D_{\gamma^{\prime}}^{\dagger}\right) \approx \operatorname{Tr}\left(D_{L} \cdots D_{2} D_{k_{2}}^{\dagger \eta_{2}} \cdots D_{k_{L}}^{\dagger} \eta_{L}\right) .
$$

The calculation of transmission amplitudes performed in Ref. 10 has now to be modified in that expressions (40) must be included. To this end, we recall the strategy devised in Refs. 7 and 10: For each encounter, one introduces coordinates on the Poincaré section adapted to the piercing by the trajectories and the linear stability of the dynamics. In encounter $\alpha$, the coordinates $\left(s_{j}^{\alpha}, u_{j}^{\alpha}\right), j=1, \ldots, l_{\alpha}-1$, describe the separation of the $(j+1)$ st piercing from the $j$ th one along the stable and unstable manifolds, respectively, of the latter. The total of $L-V$ stable and unstable coordinates are then collected in the vectors $(\mathbf{s}, \mathbf{u})$. In these coordinates, action differences of partner trajectories (approximately) read as

$$
\Delta S=S_{\gamma}-S_{\gamma^{\prime}} \approx \sum_{\alpha, j} s_{j}^{\alpha} u_{j}^{\alpha} .
$$

Moreover, the requirement that encounters be close can then be expressed in terms of the condition $\left|s_{j}^{\alpha}\right|,\left|u_{j}^{\alpha}\right| \leqslant c$ with some constant $c$, which yields the duration of an encounter,

$$
t_{\text {enc }}^{\alpha} \sim \frac{1}{\lambda} \ln \frac{c^{2}}{\max _{i}\left\{\left|s_{i}\right|\right\} \max _{j}\left\{\left|u_{j}\right|\right\}}, \quad t_{\text {enc }}^{\alpha} \rightarrow \infty .
$$

One then introduces a density $w_{T}^{\mathrm{spin}}(\mathbf{s}, \mathbf{u})$ of encounters, weighted with the spin contribution, for trajectories of duration $T$ with a given encounter structure specified by the vector $\vec{v}$. In analogy to the case without spin, ${ }^{15}$ this leads to the following approximation:

$$
\begin{aligned}
& \left\langle\sum_{\gamma} \sum_{\vec{v}} N(\vec{v}) \int_{-c}^{c} \cdots \int_{-c}^{c} d^{L-V} u d^{L-V} S\right. \\
& \left.\quad \times \exp ((i / \hbar) \Delta S) w_{T}^{\mathrm{spin}}(\mathbf{s}, \mathbf{u})\left|B_{\gamma}\right|^{2}\right\rangle_{\Delta E},
\end{aligned}
$$

to the quantity

$$
T_{a_{2} a_{1}^{\prime}}^{\mathrm{nd}}:=\left\langle\sum_{\sigma, \sigma^{\prime}=-s}^{s} \mid S_{\alpha_{2} \alpha_{1}^{\prime}}^{21}\right\rangle_{\Delta E}-\frac{2 s+1}{N_{1}+N_{2}} .
$$

After summing over all possible values of $a_{2}, a_{1}^{\prime}$, this yields the nondiagonal contribution to the energy-averaged transmission amplitude $\mathcal{T}$ [compare Eqs. (20) and (29)].

The essential point now is to calculate the density $w_{T}^{\text {spin }}(\mathbf{s}, \mathbf{u})$. In the case without spin-orbit interaction, the corresponding expression $w_{T}(\mathbf{s}, \mathbf{u})$ was defined in Ref. 7 as a density of phase-space separations $\mathbf{s}$ and $\mathbf{u}$ similar to the density $P(\varepsilon, T)$ with respect to $\varepsilon$ in the configuration-space approach. It was given as

$$
\begin{aligned}
w_{T}(\mathbf{s}, \mathbf{u})= & \frac{1}{\sum(E)} \int_{Q_{c}} d^{2} x^{\prime} \int_{\mathbb{R}^{2}} d^{2} p^{\prime} \delta\left(E-H_{0}\left(\mathbf{x}^{\prime}, \mathbf{p}^{\prime}\right)\right) \\
& \times \int_{0}^{\infty} \prod_{j=1}^{L} d t_{j} \Theta\left(T-\sum_{\alpha=1}^{V} l_{\alpha} t_{e n c}^{\alpha}-\sum_{j=1}^{L} t_{j}\right) \\
& \times \prod_{\alpha=1}^{V} \frac{1}{t_{e n c}^{\alpha}}\left[\prod_{j=2}^{l_{\alpha}} \delta\left(\left(\mathbf{X}\left(t_{\alpha j}^{\prime}\right), \mathbf{P}\left(t_{\alpha j}^{\prime}\right)\right)-z_{\alpha j}\right)\right] .
\end{aligned}
$$

The average in the first line is over all possible initial points of the trajectory. In the second line, the integration extends over all loop durations $t_{j}$; their lengths are constrained by the theta function. In order to prevent overcounting, ${ }^{7}$ the product of all encounter durations $t_{e n c}^{\alpha}$ is divided out. The last product guarantees that the position of the orbit at times when it pierces through the sections is fixed as $z_{\alpha j}$. This denotes the first point of the orbit in which it pierces through a certain 
section plus the separation thereof as specified by the coordinates $\mathbf{s}$ and $\mathbf{u}$. From Eq. (45), one obtains $w_{T}^{\text {spin }}(\mathbf{s}, \mathbf{u})$ by including $\operatorname{Tr}\left(D_{\gamma} D_{\gamma^{\prime}}^{\dagger}\right)$ under the integral. Using that the durations of encounters are semiclassically large, compare Eq. (42), the result can be obtained in analogy to Eq. (34) by employing Eq. (27). The right-hand side then yields

$$
w_{T}^{\mathrm{spin}}(\mathbf{s}, \mathbf{u}) \approx \frac{\left(T-\sum_{\alpha=1}^{V} l_{\alpha} t_{e n c}^{\alpha}\right)^{L}}{\sum(E)^{L-V} \prod_{\alpha=1}^{V} t_{e n c}^{\alpha} L !} M_{\gamma \gamma^{\prime}},
$$

i.e., a factorization into the spin-independent part identical to $w_{T}(\mathbf{s}, \mathbf{u})$ and a spin contribution,

$$
\begin{aligned}
M_{\gamma \gamma^{\prime}} & :=\int_{\mathrm{SU}(2)} \cdots \int_{\mathrm{SU}(2)} d g_{L} \cdots d g_{2} \\
& \times \operatorname{Tr}\left(\pi_{s}\left(g_{L} \cdots g_{2} g_{k_{2}}^{\eta_{2} \dagger} \cdots g_{k_{L}}^{\left.\eta_{L^{\dagger}}\right)}\right) .\right.
\end{aligned}
$$

In order to calculate Eq. (47), we follow the method devel- oped in Refs. 23 and 24 for the spectral form factor of quantum graphs with spin-orbit interaction. In analogy to Theorem 6.1 of Ref. 24, we find in the present context that

$$
M_{\gamma \gamma^{\prime}}=(2 s+1)\left(\frac{(-1)^{2 s}}{2 s+1}\right)^{L-V} .
$$

This will be proven in the Appendix. We stress that this spin contribution, apart from the spin quantum number, only depends on $L-V$.

Equation (44) can now be calculated in analogy to the case without spin. ${ }^{10}$ Starting from Eq. (43), one employs the expressions for $\Delta S$ from Eq. (41) and for $w_{T}^{\text {spin }}(\mathbf{s}, \mathbf{u})$, the sum rule from Ref. 9, and the survival probability $\rho(t)$, modified by replacing $t$ with $\left[t-\sum_{\alpha=1}^{V}\left(l_{\alpha}-1\right) t_{e n c}^{\alpha}\right]$ as in Ref. 10. This yields

$$
\begin{aligned}
T_{a_{2}, a_{1}^{\prime}}^{n d} & \approx\left\langle\frac{(2 s+1) \hbar}{m A} \sum_{\vec{v}} N(\vec{v})\left[\prod_{i=1}^{L+1} \int_{0}^{\infty} d t_{i} \exp \left(-\frac{t_{i}}{\tau}\right)\right] \int_{-c}^{c} \cdots \int_{-c}^{c} \frac{d^{L-V} u d^{L-V} S}{[\Sigma(E)]^{L-V}} \prod_{\alpha=1}^{V} \frac{\exp \left(-\frac{t_{\text {enc }}^{\alpha}+\frac{i}{\tau} \Delta S}{\hbar} \Delta S\right.}{t_{\text {enc }}^{\alpha}}\right\rangle_{\Delta E}\left(\frac{(-1)^{2 s}}{2 s+1}\right)^{L-V} \\
& \approx \frac{(2 s+1)}{N_{1}+N_{2}} \sum_{k=1}^{\infty}\left(\frac{1}{N_{1}+N_{2}}\right)^{k}\left(\frac{(-1)^{2 s}}{2 s+1}\right)^{k} \sum_{\vec{v}, L-V=k}(-1)^{V} N(\vec{v}) .
\end{aligned}
$$

The integrals over $\mathbf{s}$ and $\mathbf{u}$ were calculated in Ref. 10, and the sum over $\vec{v}$ can be carried out with the recursion formula ${ }^{10}$

$$
\sum_{\vec{v}, L-V=k}(-1)^{V} N(\vec{v})=\left(1-\frac{2}{\beta}\right)^{k}
$$

where $\beta=1$ if time-reversal symmetry is present and $\beta=2$ if time-reversal symmetry is broken.

Finally, using these results in the case of time-reversal invariance, we obtain for the full transmission matrix, including also the diagonal part,

$$
T_{a_{2}, a_{1}^{\prime}}^{\mathrm{nd}}+\frac{2 s+1}{N_{1}+N_{2}} \approx \frac{(2 s+1)^{2}}{(2 s+1)\left(N_{1}+N_{2}\right)-1},
$$

in the case of half-integer $s$, and

$$
T_{a_{2}, a_{1}^{\prime}}^{\mathrm{nd}}+\frac{2 s+1}{N_{1}+N_{2}} \approx \frac{(2 s+1)^{2}}{(2 s+1)\left(N_{1}+N_{2}\right)+1},
$$

if $s$ is integer. For $s=1 / 2$, Eq. (51) is identical with the one obtained using random matrix theory, in the circular symplectic ensemble. ${ }^{1}$
These findings can now be compared with the respective results when time reversal is absent, thus revealing the behavior of the transmission under a breaking of time reversal by, e.g., turning on a magnetic field. In that case, $\beta=2$ so that Eq. (50) vanishes, implying via Eq. (49) that only the diagonal contribution survives. The difference $\Delta \mathcal{T}=\mathcal{T}^{(\beta=1)}$ $-\mathcal{T}^{(\beta=2)}$ of the transmission coefficients is therefore

$$
\Delta \mathcal{T} \approx \frac{N_{1} N_{2}(2 s+1)}{\left(N_{1}+N_{2}\right)\left[(2 s+1)\left(N_{1}+N_{2}\right)-1\right]},
$$

in the case of half-integer $s$, and

$$
\Delta \mathcal{T} \approx \frac{-N_{1} N_{2}(2 s+1)}{\left(N_{1}+N_{2}\right)\left((2 s+1)\left(N_{1}+N_{2}\right)+1\right)},
$$

if $s$ is integer. From these expressions, one immediately concludes that the transmission (i.e., conductivity) is enhanced at zero magnetic field (when time-reversal symmetry is restored), if the spin is half-integer; thus, weak antilocalization occurs. The only semiclassical derivation of weak antilocalization so far $^{20}$ was restricted to the one-loop contribution and employed asymptotics for large $N_{1}, N_{2}$.

For integer spin, the above results predict weak localization. The latter property had previously been obtained in 
semiclassical studies where the spin-orbit interaction had been neglected. ${ }^{9}$ Of course, transport properties of bosons are different from those of fermions and relations (3) and (4) are not applicable in that case. However, expressions (52) and (54) are still measuring transport properties of, e.g., cold atoms with narrow energy distributions.

\section{SHOT NOISE}

The techniques developed above can be applied to a number of further problems arising in the context of ballistic transport through chaotic mesoscopic cavities. As a first example, we consider shot noise. To this end, one needs to compute the energy-averaged Fano factor $F$, defined as ${ }^{28}$

$$
F:=\frac{\left\langle\operatorname{Tr}\left(T T^{\dagger}-T T^{\dagger} T T^{\dagger}\right)\right\rangle_{\Delta E}}{\left\langle\operatorname{Tr}\left(T T^{\dagger}\right)\right\rangle_{\Delta E}},
$$

in terms of the transmission matrix $T=S^{21}$. The denominator has been dealt with above, and the spin-independent contribution to

$$
\operatorname{Tr}\left(T T^{\dagger} T T^{\dagger}\right)
$$

was calculated semiclassically in Ref. 15 . We are hence left with the task of determining the spin contribution to Eq. (56). Referring to the semiclassical representation [Eq. (17)], one immediately realizes that a fourfold sum over classical trajectories emerges. In addition to the case covered in Ref. 15 , each term in this sum acquires an additional factor of

$$
\operatorname{Tr}\left(D_{s}^{\dagger} D_{u} D_{v}^{\dagger} D_{w}\right),
$$

in which the indices label the trajectories involved. The diagonal contribution to the fourfold sum occurs with $s=u$ and $v=w$ or with $s=w$ and $u=v$. In both cases, unitarity implies

$$
\operatorname{Tr}\left(D_{s}^{\dagger} D_{u} D_{v}^{\dagger} D_{w}\right)=2 s+1 .
$$

Beyond this, one has to consider the encounter of four trajectories. For the first time, this has been done in quantum graphs ${ }^{14}$ and has later been extended in Ref. 15. Following the method of these papers, every trajectory consists of two parts, labeled by 1 and 2 . Approximately, one then has $s_{1}$ $=w_{1}, u_{1}=v_{1}, s_{2}=u_{2}$, and $v_{2}=w_{2}$. Thus,

$$
\operatorname{Tr}\left(D_{s}^{\dagger} D_{u} D_{v}^{\dagger} D_{w}\right) \approx \operatorname{Tr}\left(D_{s_{1}}^{\dagger} D_{s_{2}}^{\dagger} D_{s_{2}} D_{v_{1}} D_{v_{1}}^{\dagger} D_{v_{2}}^{\dagger} D_{v_{2}} D_{s_{1}}\right)=2 s+1 .
$$

Following further the calculation of the Fano factor in Ref. 15 , we obtain

$$
F \approx \frac{N_{1} N_{2}}{\left(N_{1}+N_{2}\right)^{2}},
$$

for $N_{1}, N_{2} \rightarrow \infty$. This result coincides with the respective outcome of a random matrix calculation in the symplectic ensemble to leading order as $N_{1}, N_{2} \rightarrow \infty .{ }^{1,19}$

\section{CONDUCTANCE FLUCTUATIONS}

Universality of conductance fluctuations is often characterized in terms of the energy-averaged variance of $\operatorname{Tr}\left(T T^{\dagger}\right)$. Instead of this quantity, the energy-averaged covariance of $\operatorname{Tr}\left(R^{n} R^{n \dagger}\right)$, where $n=1,2$ labels the leads, can also be considered (see Ref. 13 for details). Our calculations are based on the first paper of Ref. 13, whose method can still be applied when the Ehrenfest time is much smaller than the dwell time; this condition is fulfilled in the semiclassical limit considered here.

The calculation of the variances again involves fourfold sums over trajectories, in which the spin contribution occurs in terms of the factors

$$
\operatorname{Tr}\left(D_{s} D_{u}^{\dagger}\right) \operatorname{Tr}\left(D_{v} D_{w}^{\dagger}\right)
$$

Switching off the spin-orbit interaction while preserving the presence of spin $s$, one obtains

$$
\operatorname{Tr}\left(D_{s} D_{u}^{\dagger}\right) \operatorname{Tr}\left(D_{v} D_{w}^{\dagger}\right)=(2 s+1)^{2} .
$$

In the presence of spin-orbit interaction, one must examine the trajectories involved more closely. Here, we again consider the case $N_{1}, N_{2} \gg 1$. The trajectories are divided into three parts labeled by 1,2 , and 3 , and the relations $s_{1}=u_{1}$, $s_{2}=\bar{v}_{2}, s_{3}=u_{3}, v_{1}=w_{1}, u_{2}=\bar{w}_{2}$, and $v_{3}=w_{3}$ or $s_{1}=u_{1}, s_{2}=v_{2}$, $s_{3}=u_{3}, v_{1}=w_{1}, u_{2}=w_{2}$, and $v_{3}=w_{3}$ hold approximately. Here, an overbar indicates that these pieces are traversed in reverse direction. In the first case, this yields

$$
\operatorname{Tr}\left(D_{s} D_{u}^{\dagger}\right) \operatorname{Tr}\left(D_{v} D_{w}^{\dagger}\right) \approx \operatorname{Tr}\left(D_{s_{2}} D_{u_{2}}^{\dagger}\right) \operatorname{Tr}\left(D_{u_{2}} D_{s_{2}}^{\dagger}\right),
$$

whereas in the second case,

$$
\operatorname{Tr}\left(D_{s} D_{u}^{\dagger}\right) \operatorname{Tr}\left(D_{v} D_{w}^{\dagger}\right) \approx \operatorname{Tr}\left(D_{s_{2}} D_{u_{2}}^{\dagger}\right)^{2} .
$$

After an average over $\mathrm{SU}(2)$, very much alike in the main part of this work, we obtain for the first case $\mathrm{e}^{23,24}$

$$
\int_{\mathrm{SU}(2)} \int_{\mathrm{SU}(2)} d g_{a} d g_{b} \operatorname{Tr}\left(\pi_{s}\left(g_{a} g_{b}^{\dagger}\right)\right) \operatorname{Tr}\left(\pi_{s}\left(g_{b} g_{a}^{\dagger}\right)\right)=1,
$$

and for the second case ${ }^{21}$

$$
\int_{\mathrm{SU}(2)} \int_{\mathrm{SU}(2)} d g_{a} d g_{b}\left[\operatorname{Tr}\left(\pi_{s}\left(g_{a} g_{b}^{\dagger}\right)\right)\right]^{2}=1 .
$$

We follow Ref. 13 further and finally observe that, with $N_{1}, N_{2} \gg 1$, the energy-averaged variance of $\operatorname{Tr}\left(T T^{\dagger}\right)$ reads

$$
\left\langle\operatorname{var}\left(\operatorname{Tr}\left(T T^{\dagger}\right)\right)\right\rangle_{\Delta E} \approx 2(2 s+1)^{2} \frac{\left(N_{1} N_{2}\right)^{2}}{\left(N_{1}+N_{2}\right)^{4}},
$$

when the spin-orbit interaction is switched off, and

$$
\left\langle\operatorname{var}\left(\operatorname{Tr}\left(T T^{\dagger}\right)\right)\right\rangle_{\Delta E} \approx 2 \frac{\left(N_{1} N_{2}\right)^{2}}{\left(N_{1}+N_{2}\right)^{4}},
$$

in the presence of spin-orbit interaction. Again, this finding is in accordance with the respective leading-order result in the symplectic ensemble of RMT. ${ }^{1,19}$ 

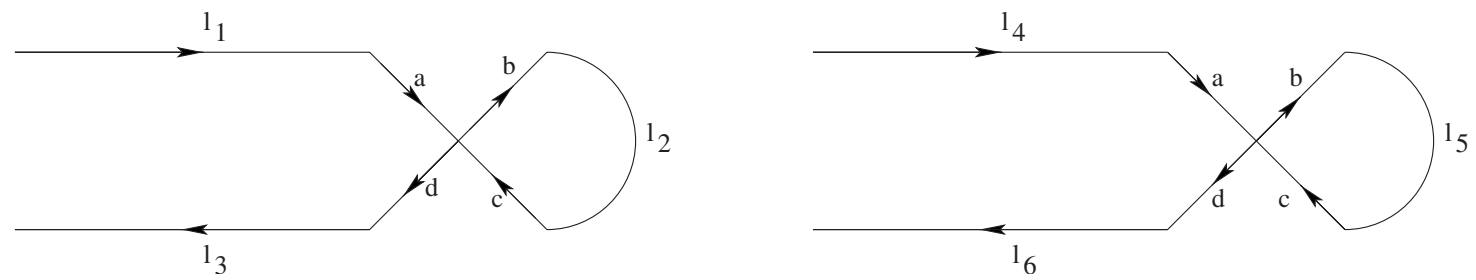

FIG. 3. Sketches of the trajectories $\gamma$ (left) and $\gamma^{\prime}$ (right) that are considered under step 2 .

\section{SUMMARY AND CONCLUSIONS}

We considered the semiclassical description of ballistic transport through chaotic mesoscopic cavities in the presence of spin-orbit interactions. Our focus was the calculation of transmission coefficients. Here, the principal task was to verify the effect of weak antilocalization in the form predicted by RMT.

Working within the framework of the Landauer formalism, our starting point was a semiclassical representation of Green's functions for Hamiltonians that contain a spin-orbit interaction. Transmission coefficients then require the evaluation of double sums over classical trajectories. The principal difficulty presented by such expressions is to get hold of the interferences thus occurring. This can be overcome successfully by exploiting the Sieber-Richter method, originally developed to perform analogous calculations in the context of spectral fluctuations in classically chaotic quantum systems.

We attacked the problem using the two established variants of the Sieber-Richter method: the configuration-space approach for the leading order and the phase-space approach for the remaining contributions. In the first case, a key input was a classical sum rule encoding an ergodic (and mixing) behavior of the combined classical spin-orbit dynamics. Essential to the success of the phase-space approach was a calculation of the spin contribution to pairs of classical trajectories that are grouped together pairwise according to the structure of their almost self-encounters. This led to the central result given in Eq. (48). The sign appearing points to the essential difference between the effects of half-integer spin as opposed to integer spin (including spin zero). This difference was then identified as responsible for weak antilocalization or localization, respectively, to occur. We finally showed

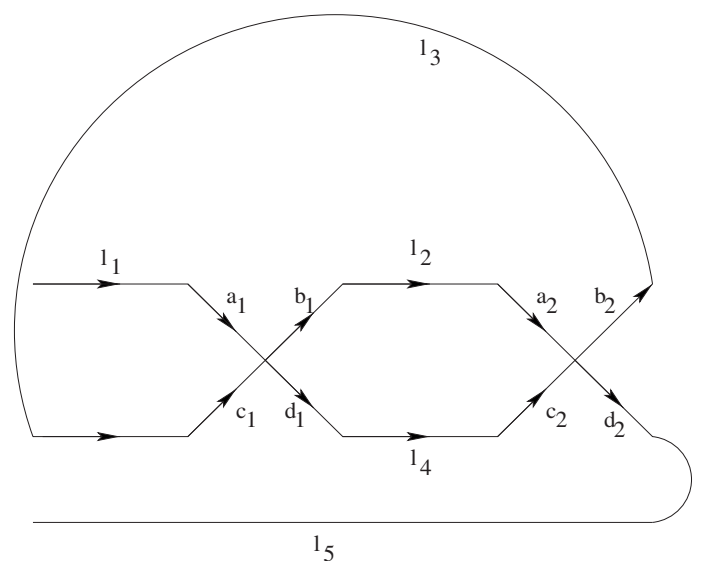

how our approach generalizes to semiclassical descriptions of shot noise and of universal conductance fluctuations.

\section{APPENDIX: PROOF OF EQUATION (48)}

We will show the validity of Eq. (48) by induction with respect to the number $n$ of 2-encounters of two trajectories $\gamma \neq \gamma^{\prime}$. The proof is based on the relations

$$
\int_{\mathrm{SU}(2)} d g \operatorname{Tr}\left(\pi_{s}(x g y g)\right)=\frac{(-1)^{2 s}}{2 s+1} \operatorname{Tr}\left(\pi_{s}\left(x y^{-1}\right)\right)
$$

and

$$
\begin{aligned}
& \int_{\mathrm{SU}(2)} \int_{\mathrm{SU}(2)} d g d h \operatorname{Tr}\left(\pi_{s}\left(g w h^{-1} x g^{-1} y h z\right)\right) \\
& =\frac{1}{(2 s+1)^{2}} \operatorname{Tr}\left(\pi_{s}(y x w z)\right),
\end{aligned}
$$

valid for all $w, x, y, z \in \mathrm{SU}(2)$. For finite groups, analogous identities have been shown in Ref. 24; their proofs can be directly carried over to the present case.

We now proceed in three steps.

(1) First, consider the case $n=0$, where $\gamma^{\prime}=\gamma$. This also means $\eta_{j}=1$ and $k_{j}=j$. Here, we obtain

$$
\begin{aligned}
M_{\gamma \gamma} & =\int_{\mathrm{SU}(2)} \cdots \int_{\mathrm{SU}(2)} d g_{L} \cdots d g_{2} \operatorname{Tr}\left(\pi_{s}\left(g_{L} \cdots g_{2} g_{2}^{\dagger} \cdots g_{L}^{\dagger}\right)\right) \\
& =2 s+1 .
\end{aligned}
$$

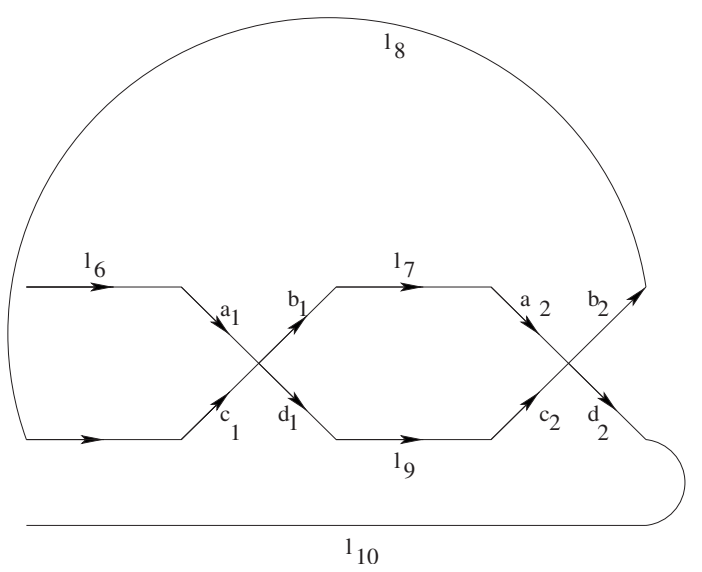

FIG. 4. Sketches of the trajectories $\gamma$ (left) and $\gamma^{\prime}$ (right) that are considered under step 3. 
(2) We assume the validity of Eq. (48) for two trajectories $\gamma=\left(l_{1}, a, b, l_{2}, c, d, l_{3}\right)$ and $\gamma^{\prime}=\left(l_{4}, a, b, l_{5}, c, d, l_{6}\right)$, as shown in Fig. 3. Here, $l_{j}$ stands for stretches of the trajectories $\gamma$ and $\gamma^{\prime}$ containing an unspecified number of 2-encounters. By assumption, the actual number of 2-encounters, where $\gamma$ differs from $\gamma^{\prime}$, is $n$. We show now that relation (48) is still valid, when we replace $\gamma^{\prime}$ with the trajectory $\gamma^{\prime \prime}=\left(l_{4}, a, \bar{c}, \bar{l}_{5}, \bar{b}, d, l_{6}\right)$. Thus, $\gamma^{\prime \prime}$ differs from $\gamma$ in $n^{\prime}=n+12$-encounters. Then,

$$
\begin{aligned}
M_{\gamma \gamma^{\prime}} & =\int_{\mathrm{SU}(2)} \cdots \int_{\mathrm{SU}(2)} d g_{a} d g_{b} d g_{c} d g_{d} \cdots \operatorname{Tr}\left(\pi_{s}\left(g_{l_{3}} g_{d} g_{c} g_{l_{2}} g_{b} g_{a} g_{l_{1}} g_{l_{4}}^{\dagger} g_{a}^{\dagger} g_{c} g_{l_{5}} g_{b} g_{d}^{\dagger} g_{l_{6}}^{\dagger}\right)\right) \\
& =\int_{\mathrm{SU}(2)} \cdots \int_{\mathrm{SU}(2)} d g_{x} d g_{y} d g_{z} \cdots \operatorname{Tr}\left(\pi_{s}\left(g_{l_{3}} g_{x} g_{l_{2}} g_{y} g_{l_{1}} g_{l_{4}}^{\dagger} g_{y}^{\dagger} g_{z} g_{l_{5}} g_{z} g_{x}^{\dagger} g_{l_{6}}^{\dagger}\right)\right) \\
& =\frac{(-1)^{2 s}}{2 s+1} \int_{\mathrm{SU}(2)} \cdots \int_{\mathrm{SU}(2)} d g_{x} d g_{y} \cdots \operatorname{Tr}\left(\pi_{s}\left(g_{l_{3}} g_{x} g_{l_{2}} g_{y} g_{l_{1}} g_{l_{4}}^{\dagger} g_{y}^{\dagger} g_{l_{5}}^{\dagger} g_{x}^{\dagger} g_{l_{6}}^{\dagger}\right)\right) \\
& =\frac{(-1)^{2 s}}{2 s+1} \int_{\mathrm{SU}(2)} \cdots \int_{\mathrm{SU}(2)} d g_{a} d g_{b} d g_{c} d g_{d} \cdots \operatorname{Tr}\left(\pi_{s}\left(g_{l_{3}} g_{d} g_{c} g_{l_{2}} g_{b} g_{a} g_{l_{1}} g_{l_{4}}^{\dagger} g_{a}^{\dagger} g_{b}^{\dagger} g_{l_{5}}^{\dagger} g_{c}^{\dagger} g_{d}^{\dagger} g_{l_{6}}^{\dagger}\right)\right) \\
& =\frac{(-1)^{2 s}}{2 s+1} M_{\gamma \gamma^{\prime}} .
\end{aligned}
$$

In the second step, we substituted $g_{d} g_{c}=g_{x}, g_{b} g_{c}=g_{z}$, and $g_{b} g_{a}=g_{y}$, and in the third one we used Eq. (A1). In the fourth step, we undid the substitution. This calculation proves that changing the number of 2-encounters, in which $\gamma$ and $\gamma^{\prime}$ differ, by 1 indeed contributes a factor of $(-1)^{2 s} /(2 s+1)$.

(3) We assume the validity of relation (48) for the two trajectories $\gamma=\left(l_{1}, a_{1}, b_{1}, l_{2}, a_{2}, b_{2}, l_{3}, c_{1}, d_{1}, l_{4}, c_{2}, d_{2}, l_{5}\right)$ and $\gamma^{\prime}=\left(l_{6}, a_{1}, b_{1}, l_{7}, a_{2}, b_{2}, l_{8}, c_{1}, d_{1}, l_{9}, c_{2}, d_{2}, l_{10}\right)$, as shown in Fig. 4. Again, we assume that the number of 2-encounters, where $\gamma$ differs from $\gamma^{\prime}$, is $n$. We then show that relation (48) is unchanged under a replacement of $\gamma^{\prime}$ with the trajectory $\gamma^{\prime}=\left(l_{6}, a_{1}, d_{1}, l_{9}, c_{2}, b_{2}, l_{8}, c_{1}, b_{1}, l_{7}, a_{2}, d_{2}, l_{10}\right)$. Notice that $\gamma^{\prime}$ cannot be constructed by applying the procedure of step 2 twice: here, the stretches $l_{6}, l_{7}$, and $l_{9}$ of $\gamma^{\prime}$ are traversed in parallel direction, whereas in step 2 the stretches $l_{4}$ and $l_{6}$ of $\gamma^{\prime}$ are traversed in antiparallel direction. A calculation similar to Eq. (A4), with the substitutions $g_{d_{j}} g_{c_{j}}=g_{x_{j}}, g_{b_{j}} g_{d_{j}}^{\dagger}=g_{z_{j}}$, and $g_{b_{j}} g_{a_{j}}$ $=g_{y_{j}}(j \in\{1,2\})$, then yields

$$
\begin{aligned}
M_{\gamma \gamma^{\prime \prime}} & =\int_{\mathrm{SU}(2)} \cdots \int_{\mathrm{SU}(2)} d g_{a_{1}} \cdots \operatorname{Tr}\left(\pi_{s}\left(g_{l_{5}} g_{d_{2}} g_{c_{2}} g_{l_{4}} g_{d_{1}} g_{c_{1}} g_{l_{3}} g_{b_{2}} g_{a_{2}} g_{l_{2}} g_{b_{1}} g_{a_{1}} g_{l_{1}} g_{l_{6}}^{\dagger} g_{a_{1}}^{\dagger} g_{d_{1}}^{\dagger} g_{l_{9}}^{\dagger} g_{c_{2}}^{\dagger} g_{b_{2}}^{\dagger} g_{l_{8}}^{\dagger} g_{c_{1}}^{\dagger} g_{b_{1}}^{\dagger} g_{l_{7}}^{\dagger} g_{a_{2}}^{\dagger} g_{d_{2}}^{\dagger} g_{l_{10}}^{\dagger}\right)\right) \\
& =\int_{\mathrm{SU}(2)} \cdots \int_{\mathrm{SU}(2)} d g_{x_{1}} \cdots \operatorname{Tr}\left(\pi_{s}\left(g_{l_{5}} g_{x_{2}} g_{l_{4}} g_{x_{1}} g_{l_{3}} g_{y_{2}} g_{l_{2}} g_{y_{1}} g_{l_{1}} g_{l_{6}}^{\dagger} g_{y_{1}}^{\dagger} g_{z_{1}} g_{l_{9}}^{\dagger} g_{x_{2}}^{\dagger} g_{z_{2}}^{\dagger} g_{l_{8}}^{\dagger} g_{x_{1}}^{\dagger} g_{z_{1}}^{\dagger} g_{l_{7}}^{\dagger} g_{y_{2}}^{\dagger} g_{z_{2}} g_{l_{10}}^{\dagger}\right)\right) \\
& =\frac{1}{(2 s+1)^{2}} \int_{\mathrm{SU}(2)} \cdots \int_{\mathrm{SU}(2)} d g_{x_{1}} d g_{y_{1}} d g_{x_{2}} d g_{y_{2}} \cdots \operatorname{Tr}\left(\pi_{s}\left(g_{l_{7}}^{\dagger} g_{y_{2}}^{\dagger} g_{l_{8}}^{\dagger} g_{x_{1}}^{\dagger} g_{l_{9}}^{\dagger} g_{x_{2}}^{\dagger} g_{l_{10}}^{\dagger} g_{l_{5}} g_{x_{2}} g_{l_{4}} g_{x_{1}} g_{l_{3}} g_{y_{2}} g_{l_{2}} g_{y_{1}} g_{l_{1}} g_{l_{6}}^{\dagger} g_{y_{1}}^{\dagger}\right)\right) \\
& =\frac{1}{(2 s+1)^{2}} M_{\gamma \gamma^{\prime}} .
\end{aligned}
$$

After these steps, Eq. (48) follows by induction because every trajectory $\gamma^{\prime}$ can be constructed successively out of $\gamma$ by using procedures of steps 2 and 3. Every $l$-encounter that does not decompose into several encounters of a lower number of trajectories (see Fig. 4 of Ref. 7 for an example) can be constructed from 2-encounters in $l-1$ steps. Every such step then brings out a factor of $(-1)^{2 s} /(2 s+1)$ in $M_{\gamma, \gamma^{\prime}}$, when this is constructed from $M_{\gamma, \gamma}=2 s+1$. Thus, $V$ encounters with $L$ stretches altogether contribute a factor $\left[(-1)^{2 s} /(2 s+1)\right]^{L-V}$, which completes the proof of Eq. (48).

*jens.bolte@uni-ulm.de

$\dagger$ daniel.waltner@physik.uni-regensburg.de

${ }^{1}$ C. W. J. Beenakker, Rev. Mod. Phys. 69, 731 (1997).

${ }^{2}$ R. Landauer, IBM J. Res. Dev. 1, 223 (1957); 32, 306 (1988).

${ }^{3}$ M. Büttiker, Phys. Rev. Lett. 57, 1761 (1986).

${ }^{4}$ H. U. Baranger, R. A. Jalabert, and A. D. Stone, Chaos 3, 665
(1993); Phys. Rev. Lett. 70, 3876 (1993).

${ }^{5}$ M. Sieber and K. Richter, Phys. Scr., T T90, 128 (2001).

${ }^{6}$ S. Müller, S. Heusler, P. Braun, F. Haake, and A. Altland, Phys. Rev. Lett. 93, 014103 (2004).

${ }^{7}$ S. Müller, S. Heusler, P. Braun, F. Haake, and A. Altland, Phys. Rev. E 72, 046207 (2005). 
${ }^{8}$ S. Heusler, S. Müller, A. Altland, P. Braun, and F. Haake, Phys. Rev. Lett. 98, 044103 (2007).

${ }^{9}$ K. Richter and M. Sieber, Phys. Rev. Lett. 89, 206801 (2002).

${ }^{10}$ S. Heusler, S. Müller, P. Braun, and F. Haake, Phys. Rev. Lett. 96, 066804 (2006).

${ }^{11} \mathrm{Ph}$. Jacquod and R. S. Whitney, Phys. Rev. B 73, 195115 (2006).

${ }^{12}$ S. Müller, S. Heusler, P. Braun, and F. Haake, New J. Phys. 9, 12 (2007).

${ }^{13}$ P. W. Brouwer and S. Rahav, arXiv:cond-mat/0512095v2 (unpublished); Phys. Rev. B 74, 075322 (2006).

${ }^{14}$ H. Schanz, M. Puhlmann, and T. Geisel, Phys. Rev. Lett. 91, 134101 (2003).

${ }^{15}$ P. Braun, S. Heusler, S. Müller, and F. Haake, J. Phys. A 39, L159 (2006).

${ }^{16}$ R. S. Whitney and Ph. Jacquod, Phys. Rev. Lett. 96, 206804 (2006).

${ }^{17}$ G. Berkolaiko, J. M. Harrison, and M. Novaes, arXiv:cond-mat/ 0703803v2 (unpublished).

${ }^{18}$ I. Žutić, J. Fabian, and S. Das Sarma, Rev. Mod. Phys. 76, 323
(2004).

${ }^{19}$ D. V. Savin and H.-J. Sommers, Phys. Rev. B 73, 081307(R) (2006).

${ }^{20}$ O. Zaitsev, D. Frustaglia, and K. Richter, Phys. Rev. Lett. 94, 026809 (2005); Phys. Rev. B 72, 155325 (2005).

${ }^{21}$ J. Bolte and S. Keppeler, Ann. Phys. (N.Y.) 274, 125 (1999).

${ }^{22}$ J. Bolte and S. Keppeler, J. Phys. A 32, 8863 (1999).

${ }^{23}$ J. Bolte and J. Harrison, J. Phys. A 36, L433 (2003).

${ }^{24} \mathrm{~J}$. Bolte and J. Harrison, in Contemporary Mathematics, edited by G. Berkolaiko, R. Carlson, S. A. Fulling, and P. Kuchment (American Mathematical Society, Providence, 2006), Vol. 415, p. 51.

${ }^{25}$ D. S. Fisher and P. A. Lee, Phys. Rev. B 23, 6851 (1981).

${ }^{26}$ H. U. Baranger and A. D. Stone, Phys. Rev. B 40, 8169 (1989).

${ }^{27}$ J. U. Nöckel, A. D. Stone, and H. U. Baranger, Phys. Rev. B 48, 17569 (1993).

${ }^{28}$ M. Büttiker, Phys. Rev. Lett. 65, 2901 (1990). 